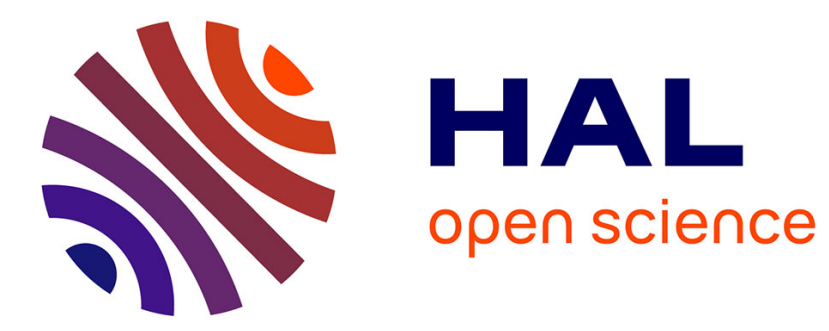

\title{
Human-robot collaboration for a shared mission
}

\author{
Abir-Beatrice Karami, Laurent Jeanpierre, Abdel-Illah Mouaddib
}

\section{To cite this version:}

Abir-Beatrice Karami, Laurent Jeanpierre, Abdel-Illah Mouaddib. Human-robot collaboration for a shared mission. 5th ACM/IEEE international conference on Human-robot interaction (HRI 10), 2010, Osaka, Japan. p155-156. hal-00969223

\section{HAL Id: hal-00969223 \\ https://hal.science/hal-00969223}

Submitted on 23 Jan 2017

HAL is a multi-disciplinary open access archive for the deposit and dissemination of scientific research documents, whether they are published or not. The documents may come from teaching and research institutions in France or abroad, or from public or private research centers.
L'archive ouverte pluridisciplinaire HAL, est destinée au dépôt et à la diffusion de documents scientifiques de niveau recherche, publiés ou non, émanant des établissements d'enseignement et de recherche français ou étrangers, des laboratoires publics ou privés.

\section{()ㅜ) $\Theta$}

Distributed under a Creative Commons Attribution - NoDerivatives| 4.0 International 


\title{
Human-Robot Collaboration for a Shared Mission
}

\author{
Abir-Beatrice Karami, Laurent Jeanpierre, Abdel-Illah Mouaddib \\ GREYC, CNRS UMR 6072 \\ Université de Caen Basse-Normandie, France \\ abir.karami,laurent,abdel-illah.mouaddib@info.unicaen.fr
}

\begin{abstract}
We are interested in collaboration domains between a robot and a human partner, the partners share a common mission without an explicit communication about their plans. The decision process of the robot agent should consider the presence of its human partner. Also, the robot planning should be flexible to human comfortability and all possible changes in the shared environment. To solve the problem of human-robot collaboration with no communication, we present a model that gives the robot the ability to build a belief over human intentions in order to predict his goals, this model counts mainly on observing the human actions. We integrate this prediction into a Partially Observable Markov Decision Process (POMDP) model to achieve the most appropriate and flexible decisions for the robot.

Index Terms-Human-Robot Collaboration, POMDP.
\end{abstract}

\section{INTRODUCTION}

In the field of robotics, the human existence for robots till lately did not exceed the purpose of serving him or asking him for information. A lot of advancements have been achieved to ease the human-robot interaction under this subject.

In [1], they present robot systems that assist old people. In those applications, robots are not really aware of the intentions of people around them, they interact with them simply if asked and only to receive information or an order for another task. The robot does not predict the points of interest of a person and adjust their behavior accordingly.

In [2], they present an assistant system to help people with dementia in completing daily life actions like washing hands. The system as an assistant recommends to the human what he should do next in case it observes a confusion or a mistake in the sequence of actions.

Recently, some research interest is increasing about applications where a robot collaborates with a human partner. In Human-Robot Collaboration the concepts of master-slave or assistant relationship exist no more. The robot and the human act jointly as equals to accomplish their mission.

Different approaches are presented for the problem of human-robot collaboration using speech and vision as a way to communicate and interact. In [3], they present a robot system that can collaborate with a human partner and also learn from him different details about the mission objects. In [4] they explain how augmented reality can help in enhancing the collaboration between the robot and the human.

In some cases, the collaboration between the robot and its human parter does not include communication. In other words, they are not able to communicate their plans to each other. The work we present explains how the robot can collaborate with a human partner without explicit communication about their plans.

\section{Human-Robot Collaboration}

Robots that are aware of their partners goals and intentions, are more likely capable to collaborate productively in order to complete the common mission. A robot during his collaboration with a human partner should be able to predict his partner's intentions. Using those information the robot should be able to make better decisions for the collaborative mission and for the comfortability of its human partner.

Human Intentions: Human behavior represents his intentions. If we see a friend entering a supermarket, we predict or assume that he is intending to do his grocery shopping. However, lack of information and false observation might lead to a bad prediction error. For example, if we do not know that this friend works for part time job in this supermarket or if we have mistaken this friend with someone that looks a lot like him, we might be led to a false prediction about this friend.

Using its observation about its human partner behavior and actions, the robot should be able to build a belief over his partner's intentions. Moreover, it should be able to update this belief each time the observations received lead to a different desire or intention in order to adapt to this change.

To be able to do that the robot needs to have some information about how to evaluate each of the human actions towards each of the possible intentions. If we suppose a mission $M=\left\{t^{t a s} k_{1}\right.$, task $\left._{2}\right\}$, knowing the human's last action $a$, we can intuitively present the robot's belief that the human is intending to accomplish any task $t a s k_{i}$ :

$$
b\left(\text { task }_{i}\right)=\frac{\text { actionvalue }\left(a, \text { task }_{i}\right)-\text { error }}{\sum_{j \in \text { tasks }} \text { actionvalue }\left(a, \text { task }_{j}\right)}
$$

\section{Collaborative Robot's System}

The environment for a robot collaborating with a human partner is partially observable, mainly because of his uncertainty about its human partner intentions, and probably some other uncertainties about other variables of the environment. This point has motivated us to represent this problem with a Partially Observable Markov Decision Process [5], knowing that POMDPs are well known for their capability of representing problems including uncertainty and providing policies that are adjustable to all possible changes in the modeled environment.

Integrating human intention in the POMDP model: By adding the human intention as a variable in the POMDP state, the robot will be able to keep track of its belief over it's human partner's intentions. Through its observations, it will be able to observe the human's behavior and his actions. At each time the 
robot acts in the environment and receives observation about the human action, it will be able to update its belief state using the transition function and the observation function.

Calculating the human action values: Following eq.1, the robot should be able to link the observed human actions with the possible human intentions. In order to do that we suggest a solution where the robot builds a number of human MDP policies, each of them includes one agent (the human) and the mission includes one task (his intention). While calculating the MDP policy, the solver calculates for each time step a value for each possible action, fig.1. Having those action values for all possible intentions (tasks), the robot can calculate a probability over all possible human intentions. We give a simple example following fig.1, if the robot receives an observation that the human last action was $a 3$, then according to the human MDP policies that the robot has calculated, it will be able to predict that most probably the human is intending to complete task 1. The reason for that is the action $a 3$ values 0.5 and 0.1 for tasks 1 and 2 respectively, which are the values that the human will accumulate if he was an agent following the MDP policy.

Human MDP for task 1

\begin{tabular}{|c|c|}
\hline human action & MDP action Value \\
\hline \hline $\mathrm{a}_{1}$ & 0.2 \\
\hline $\mathrm{a}_{2}$ & 0.3 \\
\hline $\mathrm{a}_{3}$ & 0.5 \\
\hline
\end{tabular}

Human MDP for task 2

\begin{tabular}{|c|c|}
\hline human action & MDP action Value \\
\hline \hline $\mathbf{a}_{1}$ & $\mathbf{0 . 8}$ \\
\hline $\mathbf{a}_{2}$ & $\mathbf{0 . 1}$ \\
\hline $\mathbf{a}_{3}$ & $\mathbf{0 . 1}$ \\
\hline
\end{tabular}

Fig. 1. Example of action values resulted from calculating a human MDP for each possible task.

Topological structure: Missions that are divided into subtasks give the problem a certain topological structure. For example, if we have the mission list: $M=\left\{t_{1}, t_{2}, t_{3}\right\}$, the moment the task $t_{2}$ is done by the human or the robot, the system will move to another sub-state space that represent all possibilities with the fact that task $\left(t_{2}\right)$ is done. List of missions at this moment will be $M=\left\{t_{1}, t_{3}\right\}$.

\section{ScEnARio \& EXPERIMENTAL RESUlts}

The scenario in fig. 2 consists of a robot, a human, a box and some scattered objects that should be gathered in the box. Mission is considered accomplished when all objects are put in the box. The mission can be divided into tasks; we refer to a task the fact that a robot/human intends a specific object, moves toward its position, carries it, moves toward the box position and drops it. Both the robot and the human can only be carrying one object at a moment. Once an object is carried it can not be put down elsewhere than in the box.

We have chosen two approximate POMDP solvers, and we tested results of their computed policies. The first is Forward Search Value Iteration FSVI [6] and the second is Topological Order Planning TOP [7]. We were interested in testing results from the TOP solver as our domain has a topological structure, and because of this structure, TOP solver gave better results in computation time and performance.

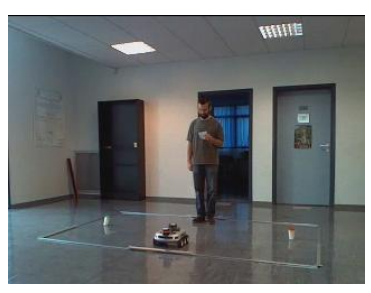

(a) Starting Mission.

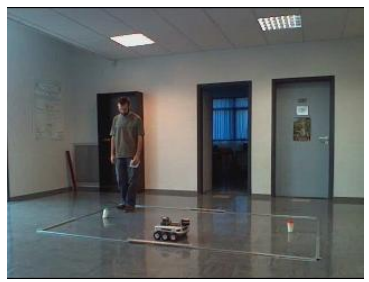

(c) Robot observes the change (d) Another change of human of human intention.

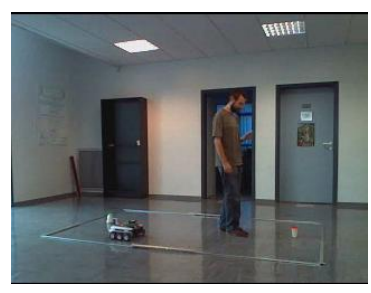

(b) The robot believe that human going to right object, it moves to left object.

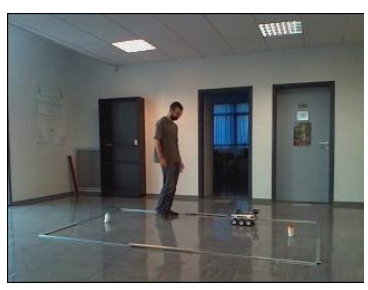

intention.
Fig. 2. Experiment with real robot. Snapshots of a video.

\section{Conclusion}

We presented a model for a collaborative robot sharing a mission with a human partner without explicit communication. We show that it is important for collaborative robots to be able to predict their human partner desires and intentions. We explained that POMDP is a very suitable model for this problem and represent a way to formalize the calculating of human intentions through observing his behavior. Results we achieved are very promising towards this problem. We noticed that the robot was successfully able to predict his partner intentions. Moreover, the robot was able to adjust rapidly to the change of the human intention, respecting the priority of the human choice that we have integrated in the reward function.

\section{REFERENCES}

[1] J. Pineau,M. Montemerlo,M. Pollack,N. Roy, and S. Thrun, Towards robotic assistants in nursing homes: Challenges and results. 2003. Special issue on Socially Interactive Robots, Robotics and Autonomous Systems.

[2] J. Boger, P. Poupart, J. Hoey, C. Boutilier, G. Fernie and A. Mihailidis, A Decision-Theoretic Approach to Task Assistance for Persons with Dementia. 2005. In Proceedings of the International Joint Conference of Artificial Intelligence IJCAI.

[3] C. Breazeal, G. Hoffman, Robots that work in collaboration with people. 2004. Proceedings of AAAI Fall Symposium on The Intersection of Cognitive Science and Robotics: From Interfaces to Intelligence. Washington $\mathrm{DC}$

[4] S.A. Green, M. Billinghurst, X. Chen, G.J. Chase, Human-Robot Collaboration: A Literature Review and Augmented Reality Approach in Design. 2008. International Journal of Advanced Robotic Systems, 5(1), pp. 1-18.

[5] A.R. Cassandra, L.P. Kaelbling and M.L. Littman, Acting Optimally in Partially Observable Stochastic Domains. 1994. Proceedings the national conference on Artificial intelligence AAAI

[6] G. Shani, R.I. Brafman and S.E. Shimony, Forward Search Value Iteration for POMDP's. 2007. In Proceedings of the International Joint Conference of Artificial Intelligence IJCAI.

[7] J.S. Dibangoye, G. Shani, B. Chaib-draa and A.I. Mouaddib, Topological Order Planner for POMDP's. 2009. In Proceedings of the International Joint Conference of Artificial Intelligence IJCAI. 